\title{
MLX Is a Transcriptional Repressor of the Mammalian Golgi Stress Response
}

\author{
Mai Taniguchi ${ }^{1^{*}}$, Kanae Sasaki-Osugi ${ }^{*}$, Masaya Oku ${ }^{2}$, Shogo Sawaguchi ${ }^{1}$, Soichiro Tanakura ${ }^{1}$, \\ Yumeto Kawai ${ }^{1}$, Sadao Wakabayashi ${ }^{1}$, and Hiderou Yoshida ${ }^{1,2^{* * *}}$ \\ ${ }^{1}$ Department of Molecular Biochemistry, Graduate School of Life Science, University of Hyogo, Hyogo \\ 678-1297, Japan, ${ }^{2}$ Department of Biophysics, Graduate School of Science, Kyoto University, Kyoto \\ 606-8502, Japan
}

\begin{abstract}
The Golgi stress response is a homeostatic mechanism that controls the capacity of the Golgi apparatus in accordance with cellular demands. When the capacity of the Golgi apparatus becomes insufficient (Golgi stress), transcription levels of Golgi-related genes encoding glycosylation enzymes, a Golgi structural protein, and components of vesicular transport are upregulated through a common cis-acting enhancer-the Golgi apparatus stress response element (GASE). Here, we identified the transcription factor MLX as a GASEbinding protein. MLX resides in the cytoplasm and does not bind to GASE in normal growth conditions, whereas MLX translocates into the nucleus and specifically binds to GASE in response to Golgi stress. Suppression of MLX expression increased transcriptional induction of target genes of the Golgi stress response, whereas overexpression of MLX reduced GASE-binding of TFE3 as well as transcriptional induction from GASE, suggesting that MLX is a transcriptional repressor of the mammalian Golgi stress response.
\end{abstract}

Key words: Golgi, stress, MLX, TFE3, GASE

\section{Introduction}

Eukaryotic cells contain a set of organelles including the endoplasmic reticulum (ER) and the Golgi apparatus, and the amount of each organelle is almost equal within a specific type of cell. When cells divide and the amount of each organelle is reduced by half, they gradually increase in amount and finally recover to their original levels. When the capacity of a certain organelle needs to be increased, cells augment its capacity in accordance with cellular demands; for instance, the endoplasmic reticulum is remarkably increased during the differentiation of plasma cells to support the synthesis of antibody proteins (Porter, 1953). These observations suggested the existence of the organelle autoregulation, which controls the amount of each organelle in accordance with cellular needs (SasakiOsugi and Yoshida, 2015). Organelle autoregulation is essential for cells to maintain the homeostasis of each organelle, although the molecular mechanisms of organelle

\footnotetext{
*These authors contributed equally to this work.

**To whom correspondence should be addressed: Hiderou Yoshida, Harima Science Garden City, Hyogo 678-1297, Japan.

Tel: +81-791-58-0212, Fax: +81-791-58-0219

E-mail: hide@sci.u-hyogo.ac.jp

Web site: http://www.sci.u-hyogo.ac.jp/life/biochem2/index-j.html
}

autoregulation have not been clarified except for the nucleus (Nurse, 2000).

Mori and Glimcher's group revealed that the ER stress response (also called the unfolded protein response) is not merely a protective mechanism to cope with stress but is also an autoregulatory mechanism that increases the capacity of the ER according to cellular demands (Iwakoshi et al., 2003; Lee et al., 2005; Reimold et al., 2001; Shaffer et al., 2004; Yoshida et al., 2001). When cells differentiate into professional secretory cells, such as plasma cells and salivary gland cells, and the function of the ER becomes insufficient to support the synthesis of large amounts of secretory proteins (ER stress), unfolded proteins accumulate in the ER and activate a homeostatic mechanism, namely, the ER stress response. In response to ER stress, ER stress sensors including pATF6(P), IRE1, and PERK are activated, and the expression levels of specific transcription factors including pATF6(N), pXBP1(S), and ATF4 are increased, resulting in transcriptional induction of ER-related genes including ER chaperone genes and finally upregulation of ER capacity (Cao and Kaufman, 2014; Gardner et al., 2013; Kimata and Kohno, 2011; Mori, 2009; Volmer and Ron, 2015; Wakabayashi and Yoshida, 2013). These studies suggested the existence of autoregulatory mechanisms in organelles other than the ER, though few 
studies have examined them in detail.

Recently, we started an analysis of the autoregulatory mechanism of the Golgi apparatus, which we named the Golgi stress response. Microarray analyses revealed that expression of Golgi-related genes encoding glycosylation enzymes (sialyltransferase 4A [SIAT4A], sialyltransferase 10 [SIAT10], fucosyltransferase 1 [FUT1] and UDP-Nacetylhexosamine pyrophosphorylase-like 1 [UAP1L1]), a Golgi-structural protein (GCP60), and components of vesicular transport (syntaxin $3 \mathrm{~A}, \mathrm{RAB} 20$, WIPI49, Giantin, and GM130) are increased when the function of the Golgi apparatus becomes insufficient (Golgi stress). Transcriptional induction of these target genes is regulated by a novel enhancer, Golgi apparatus stress response element (GASE), for which the consensus sequence is ACGTGGC (Oku et al., 2011). We isolated two basic-helix-loop-helixleucine zipper (bHLH-ZIP) transcription factors (TFE3 and MLX) as GASE-binding proteins, and revealed that TFE3 is an activator of GASE-mediated transcription. In normal growth conditions, TFE3 is phosphorylated and retained in the cytoplasm as a dormant form, whereas TFE3 is dephosphorylated and translocates into the nucleus to activate transcription of its target genes, such as SIAT4A and GCP60, upon Golgi stress (Taniguchi et al., 2015). However, the function of MLX has not been clarified.

In this study, we characterized expression and function of MLX during Golgi stress, and elucidated the molecular mechanism of the mammalian Golgi stress response.

\section{Materials and Methods}

\section{Cell culture and transfection}

Culture and transient transfection of HeLa cells were carried out as described previously (Nadanaka et al., 2008; Nave and Lemke, 1991; Uemura et al., 2009). In brief, HeLa cells were purchased from ATCC (Manassas, Virginia), and cultured in Dulbecco's modified Eagle's medium (glucose at $4.5 \mathrm{~g} /$ liter) supplemented with $10 \%$ fetal bovine serum, $2 \mathrm{mM}$ glutamate and 100 units $/ \mathrm{ml}$ penicillin and $100 \mu \mathrm{g} / \mathrm{ml}$ streptomycin. Cells were grown at $37^{\circ} \mathrm{C}$ in a humidified $5 \% \mathrm{CO}_{2}, 95 \%$ air atmosphere. For transfection of plasmids, cells were plated onto dishes at $10 \%$ confluency on day 1. On day 2 , a reporter plasmid ( $1 \mu \mathrm{g}$ of pGL3) and a reference plasmid $(0.1 \mu \mathrm{g}$ of pRL-SV40) were mixed in $1 \mathrm{x}$ HEPES-buffered saline (50 mM HEPES, $280 \mathrm{mM} \mathrm{NaCl}, 1.5 \mathrm{mM} \mathrm{Na}_{2} \mathrm{HPO}_{4}, \mathrm{pH}$ 7.08) containing $250 \mathrm{mM} \mathrm{CaCl}$ at room temperature, and then incubated with plated cells for $16 \mathrm{~h}$ at $37^{\circ} \mathrm{C}$ (Komori et al., 2012; Oku et al., 2011). After transfection, cells were washed with phosphate-buffered saline three times to remove DNA-Calcium phosphate complex, and further incubated in a fresh medium. On day 3, cells were treated with $0.3 \mu \mathrm{M}$ monensin to induce Golgi stress. On day 4, cells were harvested with $100 \mu \mathrm{l}$ of Passive Lysate Buffer (Promega). Firefly and Renilla luciferase activities were measured with $5 \mu$ of cell lysates using the Dual-Luciferase
Reporter Assay system (Promega) and CentroXS LB960 luminometer (Berthold, Wildbad, Germany). Relative activity was defined as the ratio of firefly luciferase activity to Renilla luciferase activity and was calculated by simply dividing luminescence intensity obtained with the assay for firefly luciferase by that for Renilla luciferase. For transfection of siRNA, $10 \mathrm{nM}$ siRNA mixed with Lipofectamine RNAiMAX was incubated with plated cells for $48 \mathrm{~h}$ at $37^{\circ} \mathrm{C}$ in a $\mathrm{CO}_{2}$ incubator. For transfection of yeast cells, cells were treated with plasmids in $100 \mathrm{mM}$ lithium acetate at $30^{\circ} \mathrm{C}$ for $5 \mathrm{~min}$, and then incubated in $40 \%$ polyethylene glycol at $30^{\circ} \mathrm{C}$ for $30 \mathrm{~min}$. After washed with water, cells were spread on synthetic dextrose $(\mathrm{SD})$ plates.

\section{Construction of plasmids}

Plasmids expressing human MLX were constructed by inserting a cDNA containing the entire coding region into the XhoI site of a pACT2 vector (Clontech), a pVP16 vector (Clontech), a pcDNA3.1 vector (Invitrogen) and a pCMV-MYC vector (Invitrogen). The nucleotide sequence of the small interfering RNA (siRNA) for MLX was CCGCCCUAAAGAUCAUGAAAGUGAA (siMLX-A) and AAGUGAACUAUGAGCAGAUUGUGAA (siMLX-B). For construction of MLX-DN, two highly conserved basic residues, R57A and R58S, were mutated according to a previous report (Ma et al., 2005).

\section{Yeast one-hybrid screening}

Yeast one-hybrid screening was carried out essentially as described previously (Yoshida et al., 1998) using a human pancreas cDNA library (\#HL4049AH, Clontech) and yeast strain KMY1005 (Mori et al., 1996). In brief, four tandem repeats of the GASE sequence (GCTGCGGTCCACGTGGCTTG) from the human SIAT4A promoter were placed upstream of the IRE1 promoter, driving expression of the yeast HIS3 gene and the Escherichia coli lacZ fusion gene (Fig. 1A). The reporter plasmid harboring these two reporter genes was linearized at the NcoI site present in the URA3 gene and integrated into the ura3-52 locus of yeast strain KMY1015 (MATa leu2-3, 112 ura3-52 his3-D200 trp1-D901 lys2-801 ire1D::TRP1; Ref. 10) at one copy. The resultant reporter strain (KMY1015-4xGASE) was transfected with a human pancreas cDNA library fused with the activation domain of yeast transcription factor GAL4, and incubated on SD plates lacking histidine and supplemented with $200 \mu \mathrm{M} 3-$ aminotriazole for 3 days. Human cDNAs were recovered from colonies formed on the SD plates.

\section{Preparation of anti-MLX antiserum}

A fusion protein of region [1-42] from human MLX and bacterial glutathione S-transferase (GST) was expressed in Escherichia coli and used as an antigen for the preparation of polyclonal anti-MLX antiserum in rabbits. The antiserum was purified twice through a GST-bacterial protein column and an affinity column using an antigen. 
A

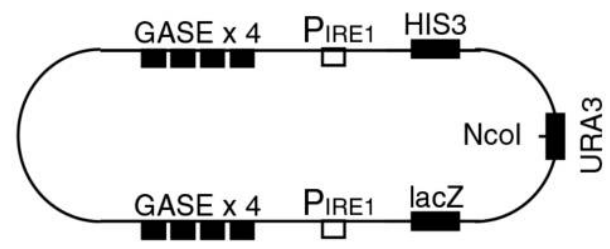

B
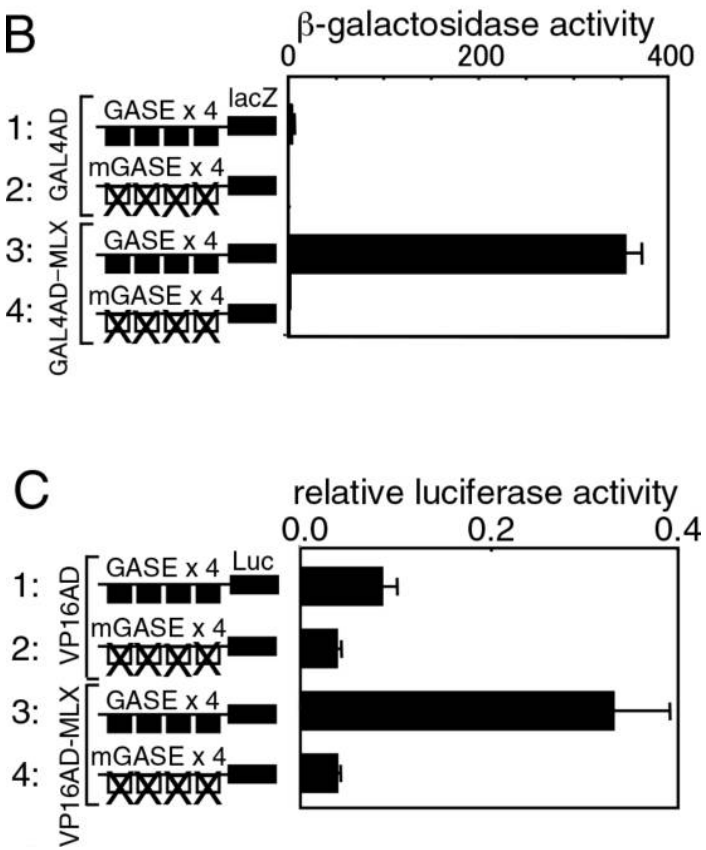

Fig. 1. Isolation of MLX as a Golgi apparatus stress response elementbinding protein by yeast one hybrid screening. (A) The reporter construct used for a yeast one-hybrid screen. To reduce basal expression, a yeast IRE1 promoter was used. The reporter plasmid was cleaved at the $N c o$ I site and integrated into the URA3 gene in the yeast genome. (B) Golgi apparatus stress response element (GASE)-binding of MLX in yeast cells. The indicated plasmids were transfected into yeast cells, and $\beta$ galactosidase activity was measured. An $\mathrm{X}$ indicates a mutant GASE sequence (catgtGC). Data from triplicate experiments are shown with standard deviations. (C) HeLa cells were transfected with the indicated plasmids and subjected to luciferase activity assays. Data from triplicate experiments are shown with standard deviations.

\section{Northern blotting, quantitative real time PCR, immunoblotting, and immunocytochemistry}

Northern blotting, quantitative real time PCR (qRT-PCR), immunoblotting, and immunocytochemistry were carried out as described previously (Taniguchi et al., 2015; Yoshida et al., 2001). For Northern blotting, total RNA prepared from HeLa cells using guanidine-phenol solution was separated on formaldehydeagarose gel. After blotting to PVDF membranes, RNA was probed with digoxigenin-labelled cDNA probes, and detected using CDPstar (Roche, Basel, Switzerland) and LAS-3000 (Fuji Film,
Japan). For qRT-PCR, total RNA was purified through NucleoSpin RNA Clean-up XS (TaKaRa, Japan), quantitated using Power SYBR Green PCR kit and Quant Studio 6 (Thermo Fisher Scientific, Waltham, Massachusetts). For immunoblotting, whole cell extracts prepared from cells were separated on SDSpolyacrylamide gels, blotted to nylon membranes and probed with primary antibodies and secondary antibodies conjugated with horse radish peroxidase. Signals were detected with luminol reagents and LAS-3000. For immunocytochemistry, cells grown on cover glasses were fixed with $2 \%$ formaldehyde, permeabilized with $0.2 \%$ Triton $\mathrm{X}-100$, and probed with primary anti-sera and secondary antisera conjugated with fluorescent dyes. Images were obtained using ECLIPSE TE2000 (Nikon, Japan) and ORCA Flash 4.0 (Hamamatsu Photonics, Japan).

\section{Chromatin immunoprecipitation assay}

Whole cell lysates were fixed with $11.1 \%$ formaldehyde, disrupted by sonication using a Bioruptor (Cosmo Bio), and immunoprecipitated using anti-MLX antiserum (Nakagawa et al., 2006). The GASE or ER stress response element (ERSE) sequences were amplified by PCR using co-precipitated genomic DNA as a template, and separated on a TAE-2\% agarose gel.

\section{Results}

\section{Isolation of a bHLH-ZIP transcription factor MLX as a GASE-binding protein}

Previously, we identified the novel enhancer element GASE, which resides in the promoters of Golgi-related genes and regulates Golgi stress-induced transcription of these genes (Oku et al., 2011). Transcription from GASE was increased upon treatment with a Golgi stress inducer (monensin), disruption of the Golgi structure (Oku et al., 2011), or insufficiency of glycosylation in the Golgi apparatus (Taniguchi et al., 2015). To identify transcription factors regulating transcriptional induction through GASE, we searched for GASE-binding proteins using a yeast onehybrid screen. The yeast HIS3 gene under the control of a GASE enhancer (GASE-HIS3 construct; Fig. 1A), as well as a human cDNA library fused with the activation domain of yeast GAL4 transcription factor (GAL4AD), was introduced into his $3 \Delta$ mutant yeast cells. If the product of a human cDNA binds to GASE and activates transcription of the HIS3 gene, the yeast cells would be able to grow on medium lacking histidine. From 3.7 million transformants, 25 positive clones were obtained. Three of the positive clones contained a plasmid expressing a transcription factor MLX.

MLX is a bHLH-ZIP transcription factor belonging to the MAX subfamily (Meroni et al., 2000). To examine whether MLX binds specifically to GASE, human MLX fused with GAL4AD (GAL4AD-MLX) was co-transfected 

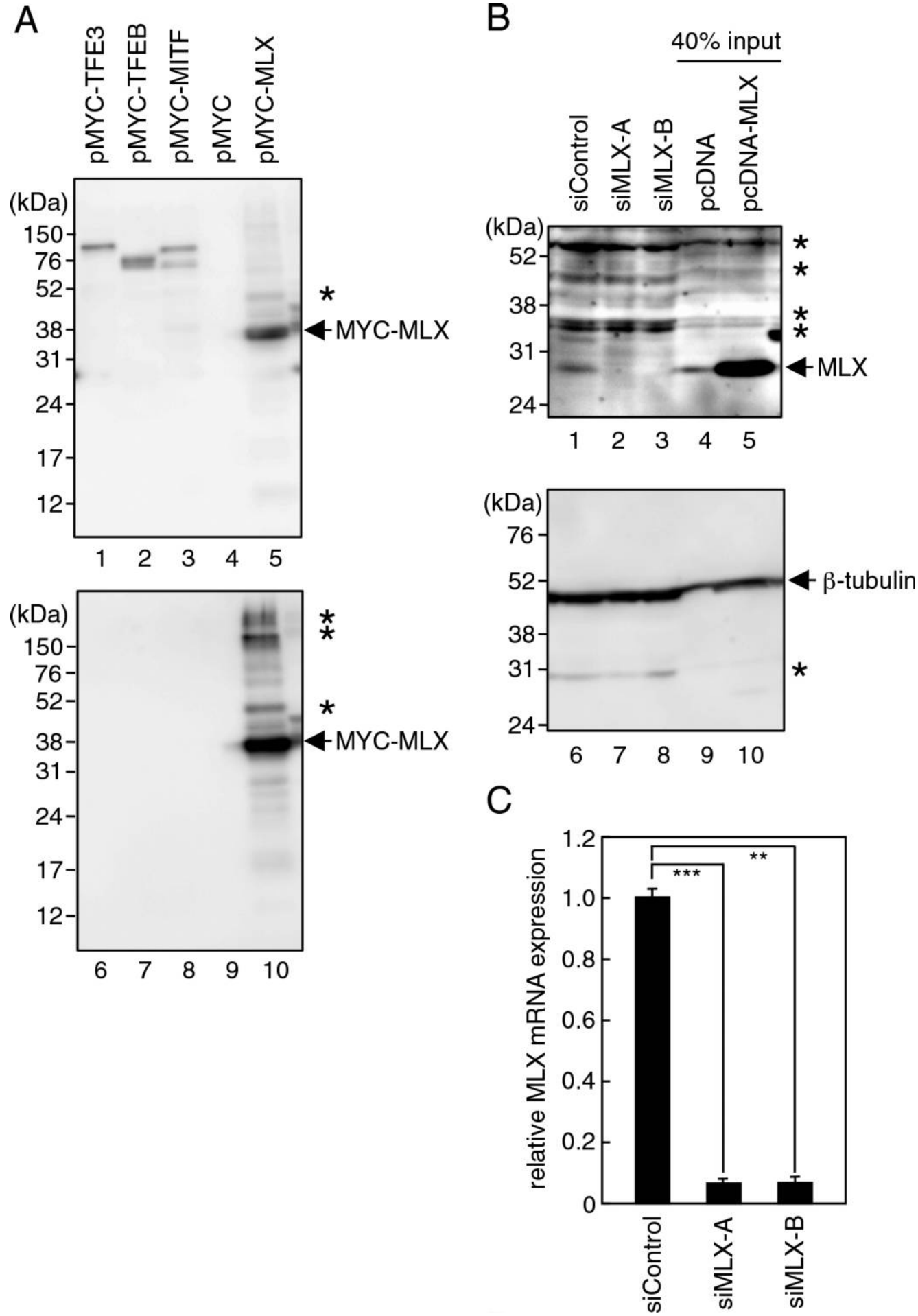

Fig. 2. Specificity of anti-MLX antiserum and suppression of MLX expression by RNA interference. (A) Quality check of the anti-MLX antiserum. Cell lysates prepared from HeLa cells transfected with the indicated plasmids were subjected to immunoblotting with an anti-MYC antibody (upper panel) or anti-MLX antiserum (lower panel). Non-specific bands are indicated by asterisks. (B) Suppression efficiency of MLX protein by small interfering RNA (siRNA) treatment. HeLa cells were transfected with the indicated siRNA or plasmid and subjected to immunoblotting probed with anti-MLX antiserum (upper panel) or anti- $\beta$ tubulin antiserum (lower panel). (C) Suppression efficiency of MLX mRNA expression by siRNAs. Expression of MLX mRNA was measured by quantitative real time PCR. Values are means $\pm \mathrm{SE}$ of two independent experiments. ***, $\mathrm{P}<0.001 ; * *, \mathrm{P}<0.01$. 
into yeast cells with a GASE-lacZ reporter construct (Fig. 1B). $\beta$-Galactosidase activity was almost negligible in cells transfected with an empty vector containing only GAL4AD (lane 1), whereas $\beta$-galactosidase activity was strongly enhanced when the cells were transfected with a plasmid expressing a GAL4AD-MLX fusion protein and a reporter construct of a wild-type GASE-lacZ construct (lane 3). Furthermore, mutation of GASE completely abolished transcriptional induction by GAL4AD-MLX (lane 4). These findings indicated that MLX binds specifically to GASE in yeast cells.

We performed similar experiments in human cells. MLX fused with the activation domain of viral transcription factor VP16 (VP16AD-MLX) was transiently expressed in HeLa cells with a reporter plasmid containing a firefly luciferase gene under the control of a GASE enhancer (GASELUC) (Oku et al., 2011) (Fig. 1C). Expression of VP16ADMLX enhanced transcription from GASE compared with expression of VP16AD alone (lanes 1 and 3), and neither affected transcription from mutant GASE (lanes 2 and 4), suggesting that MLX can bind specifically to the GASE sequence in human cells.

\section{GASE-binding of MLX is increased during Golgi stress}

To analyze the MLX protein, we generated anti-MLX antiserum (anti-MLX) and checked whether it specifically recognized MLX (Fig. 2A). Whole cell extracts prepared from HeLa cells expressing MYC-tagged MLX was subjected to immunoblotting, and we found that anti-MLX detected MYC-MLX (lower panel, lane 10), whereas it did not cross-react with either MYC-TFE3 or MYC-TFE3 family proteins (TFEB and MITF) (lanes 6-8). Expression of MYC-TFE3, TFEB, and MITF was confirmed using an anti-MYC antibody (upper panel). When expression of MLX was suppressed by siRNA against MLX (siMLX-A and siMLX-B), the band detected with the anti-MLX antiserum almost disappeared (Fig. 2B, upper panel, lanes 2 and 3), whereas the band was increased when MLX was overexpressed (lane 5), indicating that the band corresponded to endogenous MLX protein. Equal loading was confirmed using anti- $\beta$-tubulin antiserum (lower panel), and the efficiency of MLX knockdown was confirmed by qRTPCR (Fig. 2C). These findings indicated that the anti-MLX antiserum can recognize MLX specifically.

Then, we performed a chromatin immunoprecipitation (ChIP) assay with the anti-MLX antiserum to examine GASE-binding activity of endogenous MLX (Fig. 3). HeLa cells were treated with or without the Golgi stress-inducing reagent monensin, and endogenous MLX contained in cell lysates was immunoprecipitated with anti-MLX antiserum. Co-immunoprecipitated genomic DNA was used as a template for amplification of the DNA fragment containing the GASE sequence of a human SIAT4A or GCP60 promoter. In control cells, the promoter region of human SIAT4A or

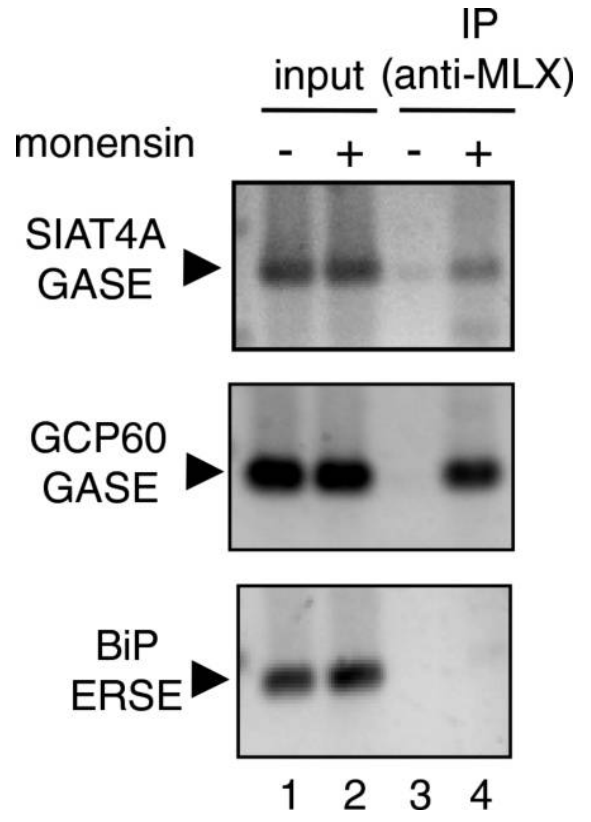

Fig. 3. Chromatin immunoprecipitation assay of MLX. MLX in cell lysates prepared from HeLa cells treated with $5 \mu \mathrm{M}$ monensin for $4 \mathrm{~h}$ was immunoprecipitated using anti-MLX antiserum, and coimmunoprecipitated Golgi apparatus stress response element (GASE) or endoplasmic reticulum stress response element (ERSE) sequences in human genomic DNA were amplified by PCR, and separated on a TAE- $2 \%$ agarose gel.

GCP60 genes including a GASE sequence was hardly amplified (upper and middle panels, lanes 3), whereas the corresponding region was specifically amplified when cells were treated with monensin (lane 4). In contrast, an ERSE sequence of the human $\mathrm{BiP}$ promoter, which is an enhancer element responsible for ER stress-induced transcription (Yoshida et al., 1998), was hardly amplified in either monensin-treated or untreated cells (lower panel, lanes 3 and 4). These findings suggested that GASE-binding by MLX is increased in response to Golgi stress.

\section{MLX translocates from the cytoplasm to the nucleus in response to Golgi stress}

Next, we examined the subcellular localization of MLX in mammalian cells. When MYC-tagged MLX was expressed in HeLa cells, MYC-MLX was found in the cytoplasm in normal growth conditions (Fig. 4, panels a, e and i). In contrast, MYC-MLX was concentrated in the nucleus after monensin treatment (panels $b-d, f-h$ and $j-1$ ). This finding suggested that MLX translocates into the nucleus in response to Golgi stress, which was consistent with results of ChIP assays in Fig. 3.

We examined MLX mRNA levels by northern blotting, and detected no change in its size or expression in response to monensin treatment (Fig. 5A). Consistent with this, the 

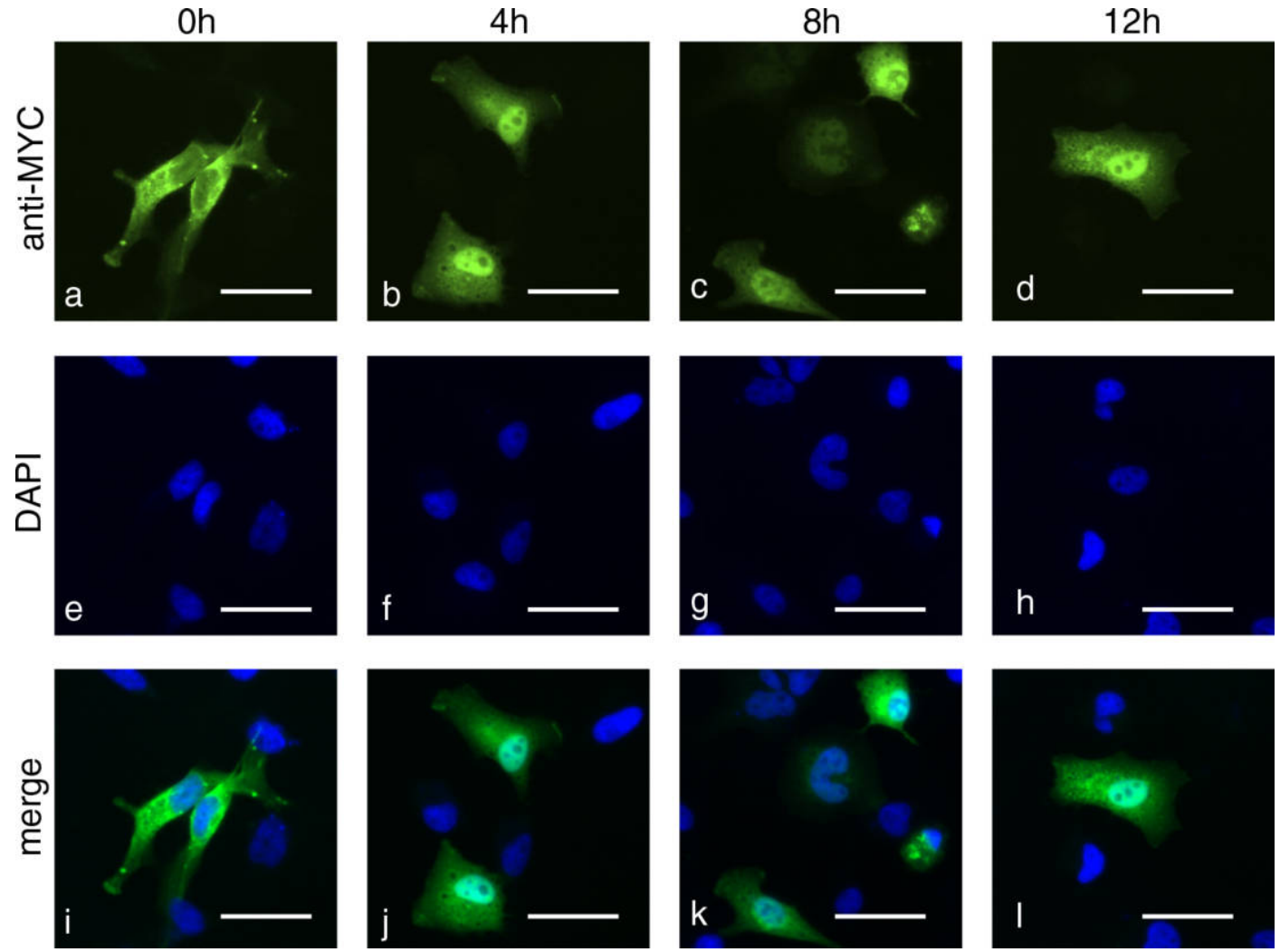

Fig. 4. Subcellular localization of MLX. HeLa cells expressing MYC-MLX were treated with $5 \mu \mathrm{M}$ monensin for the indicated periods and stained with anti-MYC antibody (upper panels) or 4',6-diamidino-2-phenylindole (DAPI; middle panel). Merged images are shown in the lower panels. Bars, $10 \mu \mathrm{m}$.

amount and electromobility of MLX protein was not changed by monensin treatment (Fig. 5B). It could be possible that unidentified escort proteins recruit MLX to the nucleus upon Golgi stress.

\section{Function of MLX in the mammalian Golgi stress response}

To examine how MLX is involved in transcriptional regulation from GASE upon Golgi stress, we evaluated the effect of MLX knockdown on GASE-mediated transcription (Fig. 6). When HeLa cells were transfected with a reporter plasmid containing a firefly luciferase gene under the control of a GASE enhancer (GASE-LUC) (Oku et al., 2011), luciferase activity was increased in response to treatment with the Golgi stress inducer monensin (lane 2), indicating that transcription from GASE was activated in response to Golgi stress. Unexpectedly, when MLX expression was suppressed by siRNA against MLX, increased luciferase activity after monensin treatment was significantly upregulated (lanes 4 and 6), suggesting that MLX is a negative regulator of GASE-mediated transcription.

Next, we examined the effect of siRNA against MLXs on the expression of endogenous target genes (SIAT4A, GCP60, and UAP1L1) during Golgi stress (Fig. 7). Expres- sion of SIAT4A, GCP60, and UAP1L1 was increased after monensin treatment (lanes 2 and 6), whereas it was significantly enhanced when MLX expression was suppressed by siRNA against MLX (lanes 4 and 8). This result was consistent with that shown in Fig. 6. We tried to knockdown both of MLX and TFE3 to reveal that transcriptional increase of these target genes by siMLX is dependent on TFE3, but unfortunately we found that such experiments are difficult because of poor knockdown efficiency. Previously, we revealed that GASE-mediated transcriptional induction was almost abolished by TFE3 knockdown (Taniguchi et al., 2015), suggesting that effect of MLX knockdown observed here is dependent on TFE3, but not on other transcription factors.

The effect of MLX overexpression was also evaluated (Fig. 8). When MLX was overexpressed in HeLa cells, transcriptional induction from GASE by monensin treatment was considerably suppressed (Fig. 8A, lane 4), whereas transcription from a GASE mutant was hardly affected (lane 8), suggesting that MLX was not a transcriptional activator but a negative regulator specific to GASE. In contrast to MLX, TFE3 is a positive regulator that binds to GASE and activates transcription upon Golgi stress (Taniguchi et al., 2015). When TFE3 was overexpressed, 


\section{A}
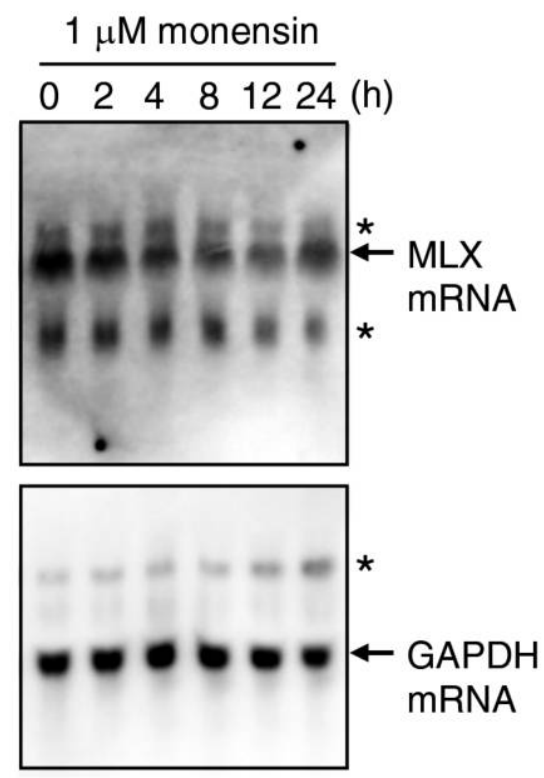

$\begin{array}{llllll}1 & 2 & 3 & 4 & 5 & 6\end{array}$
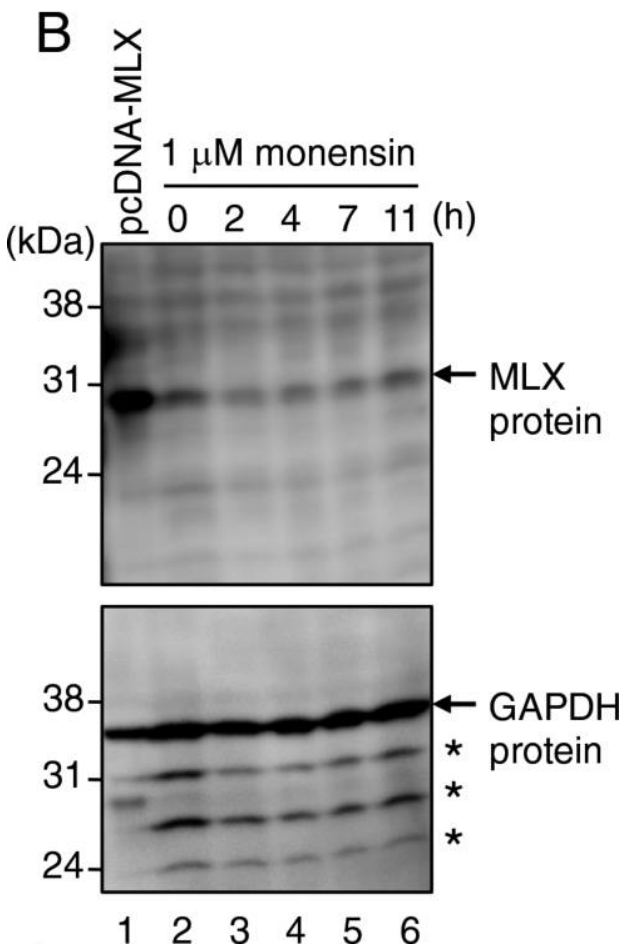

Fig. 5. Expression of MLX during Golgi stress. (A) Total RNA extracted from HeLa cells treated with $1 \mu \mathrm{M}$ monensin for the indicated periods was subjected to Northern blotting analysis using a $M L X$ cDNA probe (upper panel). The same blot was probed with a GAPDH cDNA as a loading control (lower panel). (B) Whole cell lysates prepared from HeLa cells treated with monensin were subjected to immunoblotting probed with antiMLX antiserum. The same blot was re-probed with anti-GAPDH antiserum.

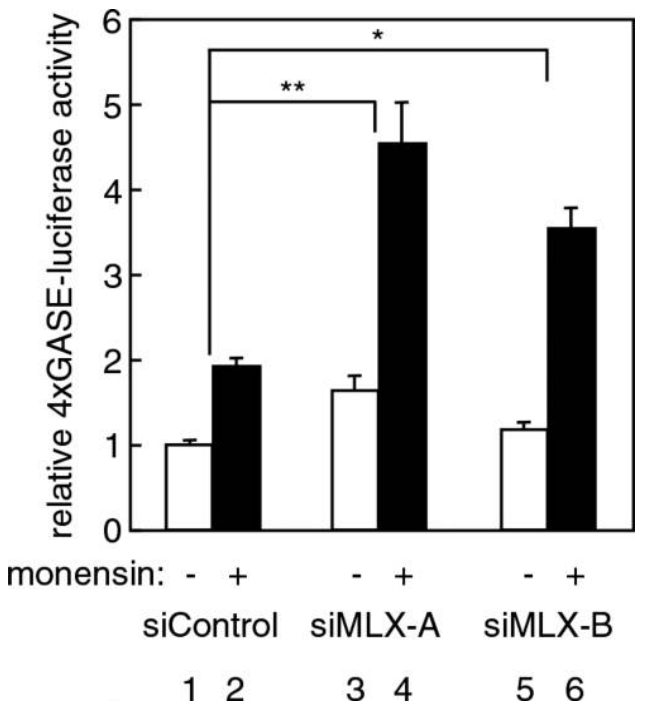

Fig. 6. Effect of suppression of MLX expression on Golgi apparatus stress response element-mediated transcriptional induction. HeLa cells transfected with the indicated small interfering RNA (siRNA) were subjected to luciferase assays with the Golgi apparatus stress response element (GASE)-LUC reporter. Open and closed bars indicate monensinuntreated and -treated cells, respectively. Values are means $\pm \mathrm{SE}$ of four independent experiments. **, $\mathrm{P}<0.01 ; *, \mathrm{P}<0.05$.

transcription from GASE was remarkably increased (Fig. 8B, lane 2), and overexpression of MLX almost abolished the positive effect of TFE3 overexpression (lane 4). We speculated that TFE3 competes with MLX for GASEbinding, and we examined this possibility using a ChIP assay (Fig. 8C). When MYC-TFE3 was overexpressed in HeLa cells, MYC-TFE3 binding to GASE was detected using a ChIP assay with an anti-MYC antibody (lane 5). As expected, when MLX was co-expressed, TFE3 binding to GASE was almost abolished (lane 6), supporting the above hypothesis. Finally, we overexpressed a dominant negative MLX mutant (MLX-DN) lacking the DNA-binding motif, which could dimerize with endogenous MLX and inhibit its DNA binding (Ma et al., 2005; Meroni et al., 2000). Overexpression of MLX-DN actually enhanced the effect of TFE3 overexpression, possibly antagonizing the negative effect of endogenous MLX (Fig. 8D, lane 4). We confirmed that expression of TFE3 was not affected by MLX or MLX-DN overexpression (Fig. 8F, left panel, lanes 4 and 6). In addition, when expression of MLX was suppressed by siMLX, transcription induction by TFE3 was significantly increased (Fig. 8E, lanes 4 and 6). Transcription was not remarkably induced when only MLX-DN or siMLX was overexpressed without co-expression of TFE3 (Fig. $8 \mathrm{D}$, lane 3 and Fig. 8E, lanes 3 and 5), because most of TFE3 molecules were retained in the cytoplasm as an inactive form and were not able to bind to GASE even in the absence of MLX. Moreover, we investigated whether MLX can form a heterodimer with TFE3 by co- 
A
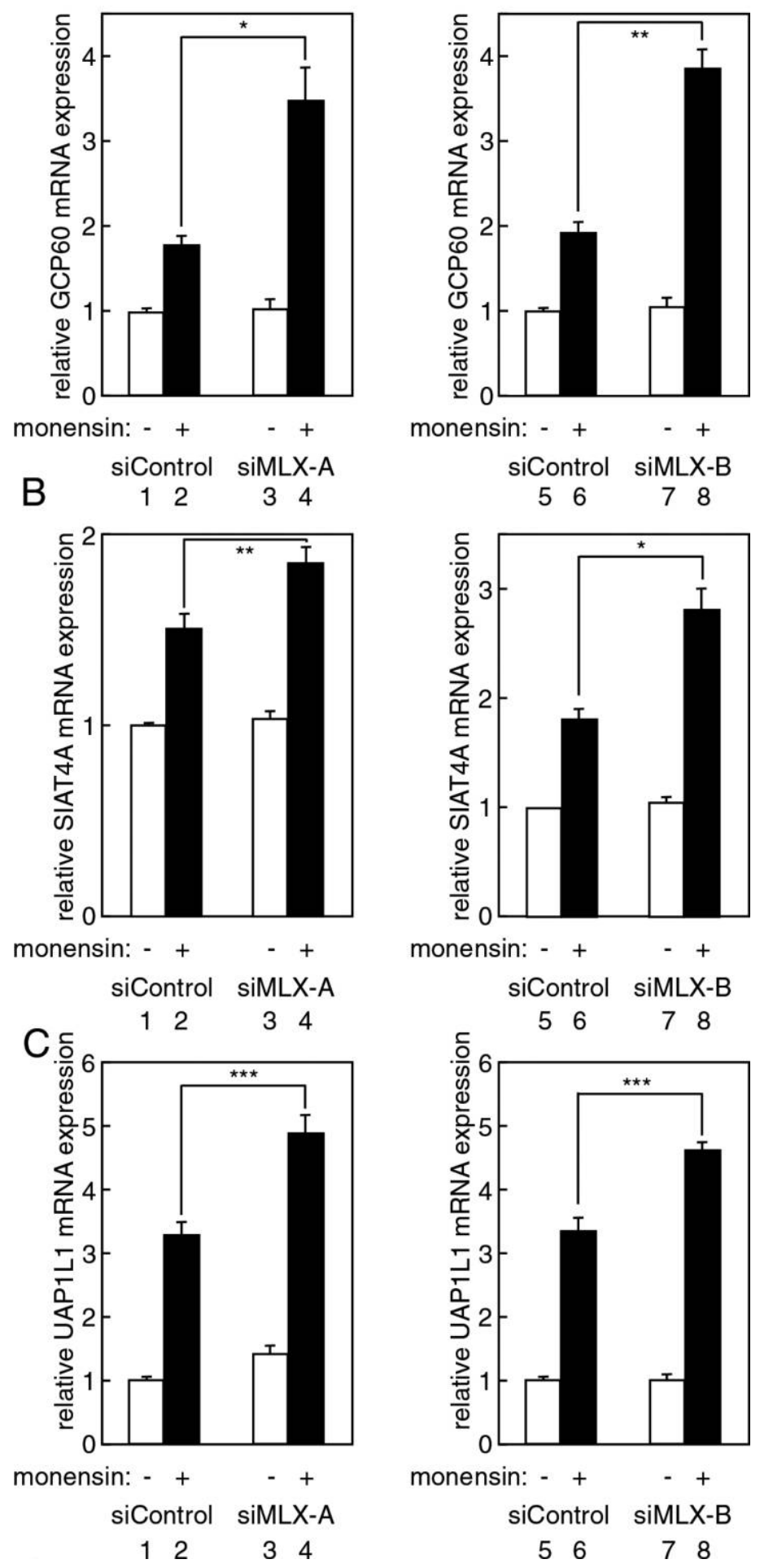

Fig. 7. Effect of suppression of MLX expression on transcriptional induction of Golgi-related genes. Total RNA prepared from HeLa cells transfected with the indicated small interfering RNA (siRNA) was subjected to quantitative real time PCR to measure expression level of (A) SIAT4A, (B) GCP60, and (C) UAP1L1 mRNA. Open and closed bars indicate monensin-untreated and -treated cells, respectively. Values are means \pm SE of three independent experiments. ***, $\mathrm{P}<0.001$; **, $\mathrm{P}<0.01$; *, $\mathrm{P}<0.05$. 

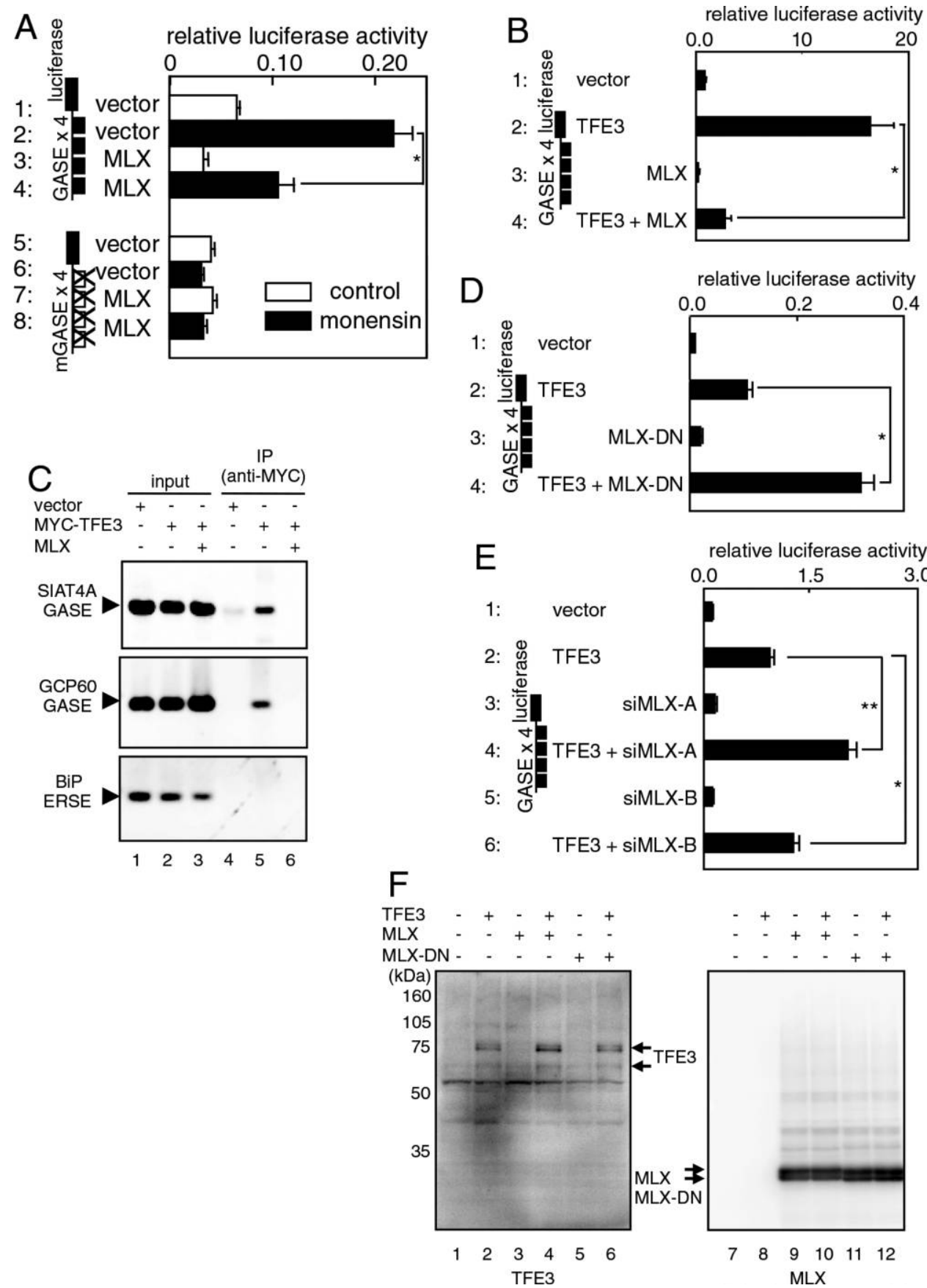

Fig. 8. Effect of MLX overexpression on transcriptional induction of Golgi-related genes. (A) HeLa cells transfected with the indicated Golgi apparatus stress response element (GASE)-LUC reporter and MLX expression plasmids were treated with $0.3 \mu \mathrm{M}$ monensin for $16 \mathrm{~h}$ and then subjected to luciferase assays. Values are means \pm SD of three independent experiments. (B) HeLa cells were transfected with TFE3 or MLX expression plasmids as well as the GASE-LUC reporter and subjected to luciferase assays. Values are means \pm SE of three independent experiments. (C) Whole cell lysates prepared from HeLa cells transfected with the expression plasmids for MYC-TFE3 and MLX were subjected to chromatin immunoprecipitation using an anti-MYC antibody and indicated primers, as described in Fig. 3. (D) HeLa cells were transfected with the GASE-LUC reporter plasmid and expression plasmids for TFE3 and MLX-DN and subjected to luciferase assays. Values are means \pm SD of three independent experiments. (E) HeLa cells were transfected with the GASE-LUC reporter plasmid, an expression plasmid for TFE3 and siMLXs and subjected to luciferase assays. Values are means \pm SE of four independent experiments. (F) Expression levels of TFE3, MLX, and MLX-DN in (A)-(D) were checked by immunoblotting with anti-TFE3 (left panel) and anti-MLX antisera (right panel). 

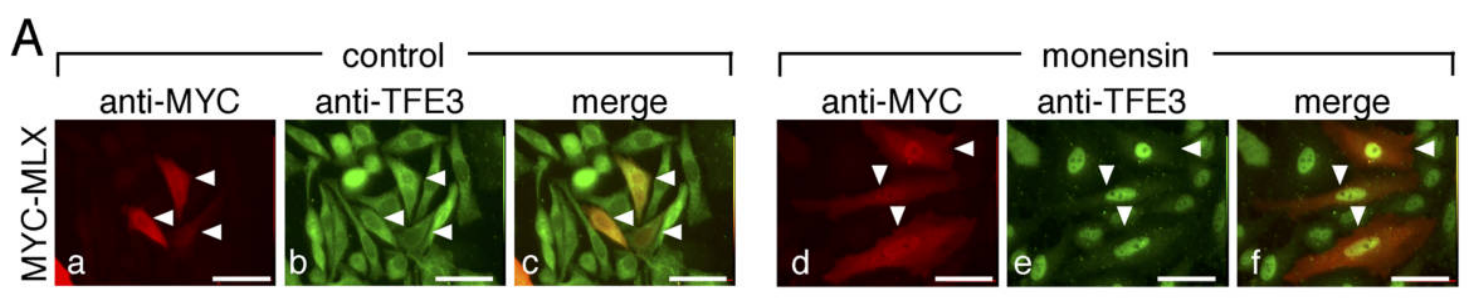

\section{B}
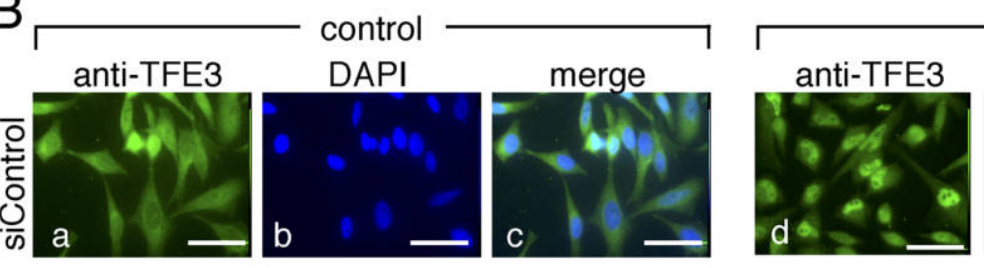

monensin
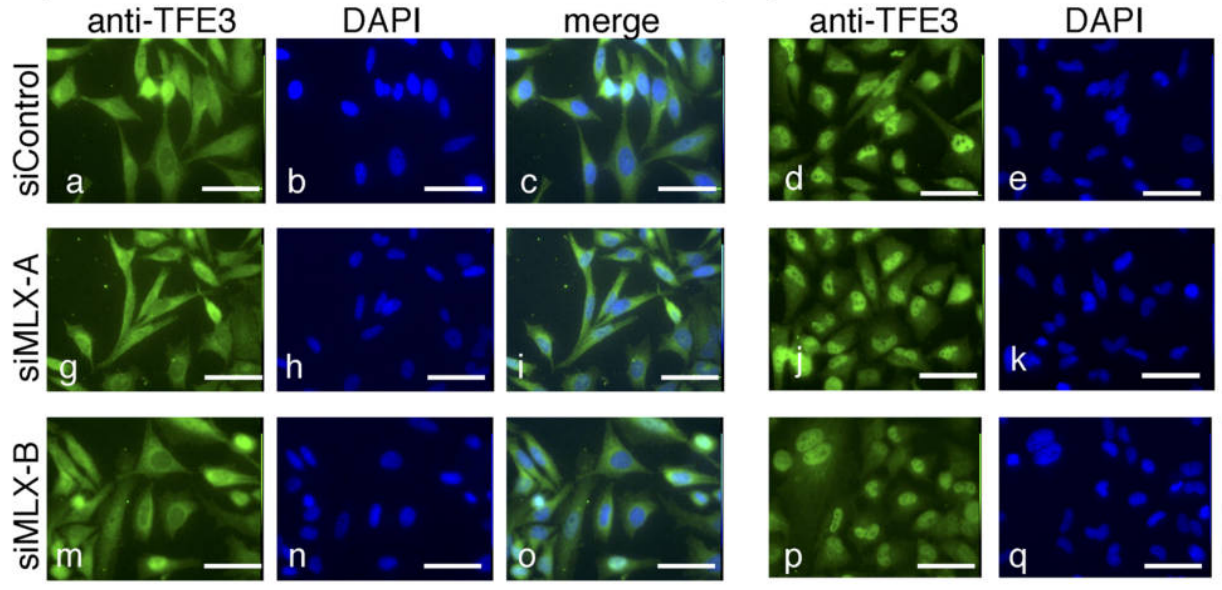

merge
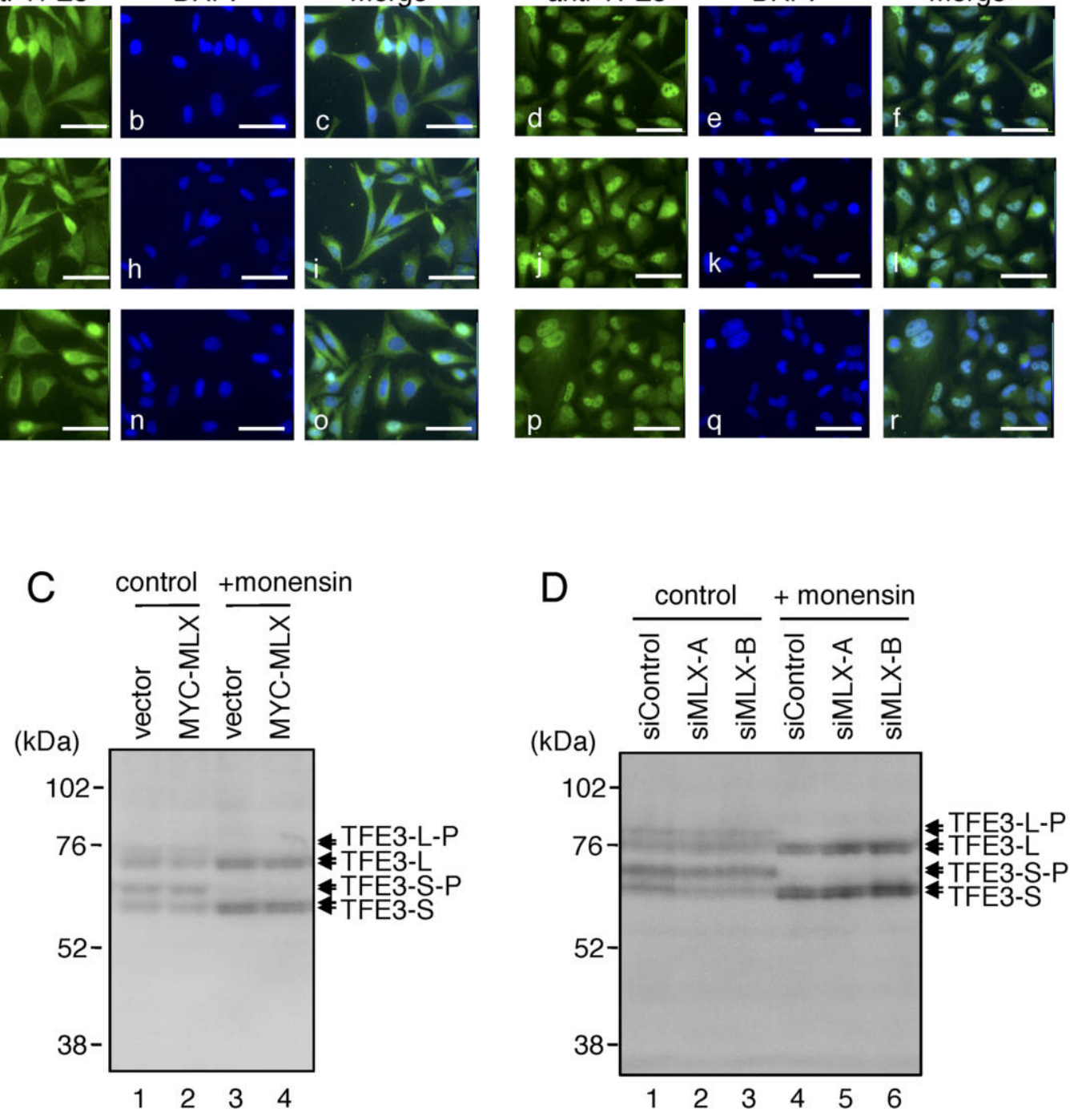

Fig. 9. Effect of MLX overexpression and siMLX on subcellular localization and phosphorylation status of TFE3. (A) HeLa cells transfected with a MYC-MLX expression vector were stained with anti-MYC and anti-TFE3 antisera. Cells were treated or untreated with $5 \mu \mathrm{M}$ monensin for $3 \mathrm{~h}$. Cells overexpressing MYC-MLX were indicated by arrow heads. Bars, $10 \mu \mathrm{m}$. (B) HeLa cells transfected with siControl, siMLX-A or siMLX-B were treated with $5 \mu \mathrm{M}$ monensin for $3 \mathrm{~h}$, and stained with anti-TFE3 antiserum. Bars, $10 \mu \mathrm{m}$. (C and D) Whole cell extracts prepared from cells transfected with a MYC-MLX expression vector (C) or siMLX (D) were subjected to immunoblotting with anti-TFE3 antiserum. Positions of much (TFE3-L-P and TFE3-SP) or less phosphorylated TFE3 (TFE3-L and TFE3-S) were indicated by arrows. TFE3-L and TFE3-S indicate long and short forms of TFE3, respectively. 
immunoprecipitation and two-hybrid assays in HeLa cells, but their heterodimerization was not be detected (data not shown). From these observations, we concluded that MLX is a negative regulator of GASE-mediated transcription in the mammalian Golgi stress response.

Since it is possible that MLX overexpression and siMLX affect subcellular localization and phosphorylation status of TFE3, we checked this possibility (Fig. 9). When MYCMLX was overexpressed in HeLa cells treated or untreated with monensin, we observed no difference of subcellular localization of TFE3 (Fig. 9A). In addition, Suppression of MLX expression by siRNA also caused no change of subcellular localization of TFE3 (Fig. 9B). Moreover, we investigated phosphorylation status of TFE3 in these cells, and found that neither MLX overexpression nor MLX knockdown affected phosphorylation status of TFE3 (Fig. 9C and 9D). These suggested that MLX affects GASEmediated transcriptional induction by TFE3, not through changing subcellular localization or phosphorylation status of TFE 3 but through competitive inhibition of GASE binding of TFE3.

\section{Discussion}

Here, we identified a transcription factor, MLX, as a GASE-binding protein. In normal growth conditions, MLX is located in the cytoplasm, whereas it localized to the nucleus and bound to GASE in response to Golgi stress. Suppression of MLX expression resulted in increased transcription from GASE upon Golgi stress, whereas its overexpression attenuated GASE binding of TFE3 and GASEmediated transcriptional induction, indicating that MLX negatively regulates Golgi stress-induced transcription. A dominant negative mutant of MLX attenuated MLX activity but rather enhanced TFE3 activity, suggesting that MLX forms a homodimer, whereas MLX cannot form a heterodimer with TFE3. These findings greatly advance our understanding of the mammalian Golgi stress response.

MLX was first identified as a protein interacting with transcription factor Mad (Meroni et al., 2000). Mad forms a heterodimer with Max and opposes the growth-promoting action of MYC-Max heterodimers (Billin and Ayer, 2006). MLX also forms a heterodimer with MondoA or ChREBP/ MondoB, which are involved in transcriptional induction of lipogenic genes in response to glucose (Billin and Ayer, 2006). ChREBP is the direct target of glucose signaling: upon activation by glucose signaling, ChREBP is dephosphorylated and translocates into the nucleus (Uyeda and Repa, 2006). MLX serves to help recruit ChREBP to the promoters of lipogenic genes, because a homodimer of ChREBP cannot bind to the carbohydrate response element (ChRE), though MLX does not contain a transcriptional activation domain. From these findings, it was suggested that MLX is a partner protein of various bHLH-ZIP tran-
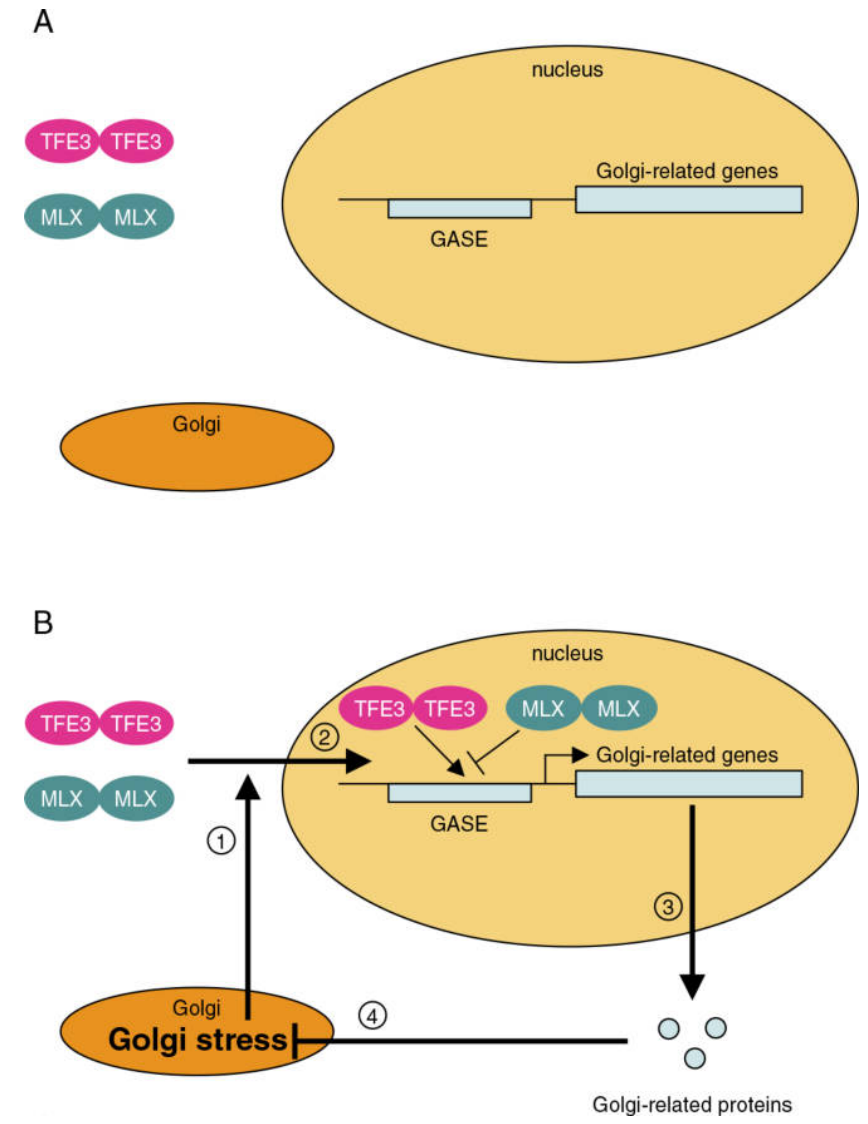

Fig. 10. Working hypothesis of MLX function in the mammalian Golgi stress response. Details are described in the text.

scription factors, including Mad, Max, ChREBP, and MondoA, and is involved in diverse biological phenomena. In the case of the mammalian Golgi stress response, MLX may function without forming a heterodimer with another unidentified transcription factor to suppress transcription from GASE, because overexpression of MLX was sufficient for transcriptional suppression (Fig. 8).

It is not unusual that several transcription factors that belong to the same gene family coordinately regulate transcription. cAMP-response element binding protein (CREB) and its related transcription factor cAMP response element modulator (CREM) share a similar structure, and CREB is a transcriptional activator whereas some splicing variants of CREM lacking the transactivation domain (S-CREM and ICER) are transcriptional repressors (Lonze and Ginty, 2002). Like S-CREM and ICER, MLX competitively binds to GASE, prevents TFE3 from GASE-binding, and represses the transcriptional induction of Golgi-related genes during Golgi stress.

In the case of the mammalian ER stress response, transcriptional induction of ER chaperone genes in response to ER stress is regulated by two transcription factors, ATF6 $\alpha$ and ATF6 $\beta$. These transcription factors are encoded by sep- 
arate genes and share similar structures. ATF6 $\alpha$ is a highly active transcriptional activator, whereas ATF6 $\beta$ is less active. These two similar transcription factors jointly regulate transcriptional induction upon ER stress by binding to the same enhancer element ERSE.

Fig. 10 shows our current working hypothesis. In normal growth conditions, MLX and TFE3 are retained in the cytoplasm in dormant forms (Fig. 10A). Upon Golgi stress, MLX and TFE3 are recruited to the nucleus, competitively bind to GASE and suppress and activate the transcription of Golgi-related genes, respectively (Fig. 10B). The regulatory mechanism of MLX nuclear translocation upon Golgi stress could be one of the key steps in the mammalian Golgi stress response, and its identification is an interesting future subject.

Acknowledgments. We thank Dr. Kazutoshi Mori for providing the yeast one-hybrid system, and Ms. Mikiko Ochiai for secretarial assistance. This work was supported by MEXT and JSPS KAKENHI (Grant Number 25650070, 16K07356 and 15J05492) and a Grant-in-Aid for Scientific Research on Innovative Areas of MEXT (No. 23110007).

\section{References}

Billin, A.N. and Ayer, D.E. 2006. The Mlx network: evidence for a parallel Max-like transcriptional network that regulates energy metabolism. Curr. Top. Microbiol. Immunol., 302: 255-278.

Cao, S.S. and Kaufman, R.J. 2014. Endoplasmic reticulum stress and oxidative stress in cell fate decision and human disease. Antioxid. Redox Signal., 21: 396-413.

Gardner, B.M., Pincus, D., Gotthardt, K., Gallagher, C.M., and Walter, P. 2013. Endoplasmic reticulum stress sensing in the unfolded protein response. Cold Spring Harb. Perspect. Biol., 5: a013169.

Iwakoshi, N.N., Lee, A.H., Vallabhajosyula, P., Otipoby, K.L., Rajewsky, K., and Glimcher, L.H. 2003. Plasma cell differentiation and the unfolded protein response intersect at the transcription factor XBP-1. Nat. Immunol., 4: 321-329.

Kimata, Y. and Kohno, K. 2011. Endoplasmic reticulum stress-sensing mechanisms in yeast and mammalian cells. Curr. Opin. Cell Biol., 23: 135-142.

Komori, R., Taniguchi, M., Ichikawa, Y., Uemura, A., Oku, M., Wakabayashi, S., Higuchi, K., and Yoshida, H. 2012. Ultraviolet a induces endoplasmic reticulum stress response in human dermal fibroblasts. Cell Struct. Funct., 37: 49-53.

Lee, A.H., Chu, G.C., Iwakoshi, N.N., and Glimcher, L.H. 2005. XBP-1 is required for biogenesis of cellular secretory machinery of exocrine glands. $E M B O$ J., 24: 4368-4380.

Lonze, B.E. and Ginty, D.D. 2002. Function and regulation of CREB family transcription factors in the nervous system. Neuron, 35: 605-623.

Ma, L., Tsatsos, N.G., and Towle, H.C. 2005. Direct role of ChREBP.Mlx in regulating hepatic glucose-responsive genes. J. Biol. Chem., 280: 12019-12027.

Meroni, G., Cairo, S., Merla, G., Messali, S., Brent, R., Ballabio, A., and Reymond, A. 2000. Mlx, a new Max-like bHLHZip family member: the center stage of a novel transcription factors regulatory pathway? Oncogene, 19: 3266-3277.

Mori, K., Kawahara, T., Yoshida, H., Yanagi, H., and Yura, T. 1996. Signalling from endoplasmic reticulum to nucleus: transcription factor with a basic-leucine zipper motif is required for the unfolded proteinresponse pathway. Genes Cells, 1: 803-817.

Mori, K. 2009. Signalling pathways in the unfolded protein response: development from yeast to mammals. J. Biochem., 146: 743-750.

Nadanaka, S., Ishida, M., Ikegami, M., and Kitagawa, H. 2008. Chondroitin 4-O-sulfotransferase-1 modulates Wnt-3a signaling through control of E disaccharide expression of chondroitin sulfate. J. Biol. Chem., 283: 27333-27343.

Nakagawa, Y., Shimano, H., Yoshikawa, T., Ide, T., Tamura, M., Furusawa, M., Yamamoto, T., Inoue, N., Matsuzaka, T., Takahashi, A., Hasty, A.H., Suzuki, H., Sone, H., Toyoshima, H., Yahagi, N., and Yamada, N. 2006. TFE3 transcriptionally activates hepatic IRS-2, participates in insulin signaling and ameliorates diabetes. Nat. Med., 12: 107-113.

Nave, K.A. and Lemke, G. 1991. Induction of the myelin proteolipid protein (PLP) gene in C6 glioblastoma cells: functional analysis of the PLP promotor. J. Neurosci., 11: 3060-3069.

Nurse, P. 2000. A long twentieth century of the cell cycle and beyond. Cell, 100: 71-78.

Oku, M., Tanakura, S., Uemura, A., Sohda, M., Misumi, Y., Taniguchi, M., Wakabayashi, S., and Yoshida, H. 2011. Novel cis-acting element GASE regulates transcriptional induction by the Golgi stress response. Cell Struct. Funct., 36: 1-12.

Porter, K.R. 1953. Observations on a submicroscopic basophilic component of cytoplasm. J. Exp. Med., 97: 727-750.

Reimold, A.M., Iwakoshi, N.N., Manis, J., Vallabhajosyula, P., Szomolanyi-Tsuda, E., Gravallese, E.M., Friend, D., Grusby, M.J., Alt, F., and Glimcher, L.H. 2001. Plasma cell differentiation requires the transcription factor XBP-1. Nature, 412: 300-307.

Sasaki-Osugi, K. and Yoshida, H. 2015. Organelle autoregulation-stress responses in the ER, Golgi, mitochondria and lysosome. J. Biochem., in press.

Shaffer, A.L., Shapiro-Shelef, M., Iwakoshi, N.N., Lee, A.H., Qian, S.B., Zhao, H., Yu, X., Yang, L., Tan, B.K., Rosenwald, A., Hurt, E.M., Petroulakis, E., Sonenberg, N., Yewdell, J.W., Calame, K., Glimcher, L.H., and Staudt, L.M. 2004. XBP1, downstream of Blimp-1, expands the secretory apparatus and other organelles, and increases protein synthesis in plasma cell differentiation. Immunity, 21: 81-93.

Taniguchi, M., Nadanaka, S., Tanakura, S., Sawaguchi, S., Midori, S., Kawai, Y., Yamaguchi, S., Shimada, Y., Nakamura, Y., Matsumura, Y., Fujita, N., Araki, N., Yamamoto, M., Oku, M., Wakabayashi, S., Kitagawa, H., and Yoshida, H. 2015. TFE3 Is a bHLH-ZIP-type Transcription Factor that Regulates the Mammalian Golgi Stress Response. Cell Struct. Funct., 40: 13-30.

Uemura, A., Oku, M., Mori, K., and Yoshida, H. 2009. Unconventional splicing of XBP1 mRNA occurs in the cytoplasm during the mammalian unfolded protein response. J. Cell Sci., 122: 2877-2886.

Uyeda, K. and Repa, J.J. 2006. Carbohydrate response element binding protein, ChREBP, a transcription factor coupling hepatic glucose utilization and lipid synthesis. Cell Metab., 4: 107-110.

Volmer, R. and Ron, D. 2015. Lipid-dependent regulation of the unfolded protein response. Curr. Opin. Cell Biol., 33: 67-73.

Wakabayashi, S. and Yoshida, H. 2013. The essential biology of the endoplasmic reticulum stress response for structural and computational biologists. Comput. Struct. Biotechnol. J., 6: e201303010.

Yoshida, H., Haze, K., Yanagi, H., Yura, T., and Mori, K. 1998. Identification of the cis-acting endoplasmic reticulum stress response element responsible for transcriptional induction of mammalian glucoseregulated proteins. Involvement of basic leucine zipper transcription factors. J. Biol. Chem., 273: 33741-33749.

Yoshida, H., Matsui, T., Yamamoto, A., Okada, T., and Mori, K. 2001. XBP1 mRNA is induced by ATF6 and spliced by IRE1 in response to ER stress to produce a highly active transcription factor. Cell, 107: 881-891.

(Received for publication, March 11, 2016, accepted, May 27, 2016 and published online, June 2, 2016) 